\title{
Neck Circumference as an Independent Cardiometabolic Risk Factor: A Cross-sectional Study in Kinshasa
}

\author{
Danny Mafuta-Munganga ${ }^{1}$, Benjamin Longo-Mbenza ${ }^{1,2,3}$, Gedeon Longo-Longo ${ }^{2}$, Manzala $^{2}$, \\ Victor Nzuzi ${ }^{2}$, Jean Bosco Kasiam Lasi On'kin ${ }^{1}$, Etienne Mokondjimobe ${ }^{2,4}$, \\ Aliocha Nkondila Natuhoyila ${ }^{2, *}$ \\ ${ }^{1}$ Department of Internal Medicine, University of Kinshasa, Kinshasa, Democratic Republic of Congo \\ ${ }^{2}$ Department of Public Health, Lomo University of Research, Kinshasa, Democratic Republic of Congo \\ ${ }^{3}$ Department of Environments, Faculty of Health Sciences, Walter Sisulu University, Mthatha, South Africa \\ ${ }^{4}$ Department of Environments, Faculty of Health Sciences, Marien Ngouabi University, Brazzaville, Republic of Congo
}

Email address:

nkodilaaliocha@gmail.com (A. N. Natuhoyila)

${ }^{*}$ Corresponding author

\section{To cite this article:}

Danny Mafuta-Munganga, Benjamin Longo-Mbenza, Gedeon Longo-Longo, Manzala, Victor Nzuzi, Jean Bosco Kasiam Lasi On'kin, Etienne Mokondjimobe, Aliocha Nkondila Natuhoyila. Neck Circumference as an Independent Cardiometabolic Risk Factor: A Crosssectional Study in Kinshasa. International Journal of Diabetes and Endocrinology. Vol. 5, No. 2, 2020, pp. 27-33. doi: $10.11648 /$ j.ijde.20200502.13

Received: June 24, 2020; Accepted: July 16, 2020; Published: August 13, 2020

\begin{abstract}
Objective: To investigate the association between neck circumference (NC) and traditional cardiometabolic risk factors (CMRF) among adult's population at Kinshasa. Methods: A total of 400 participants were recruited. Spearman's correlation coefficient was employed to test the correlations between NC and CMRF. The association of NC with CMRF (dependent variables) was assessed by logistic regression. The receiver operating characteristics (ROC) curve analysis had allowed determining the cut-off points of NC to detect the presence of CMRF. Results: The average of age and WC was $55.4 \pm 12.0$ years and $79.8 \pm 12.0 \mathrm{~cm}$, respectively. The median value of BMI was significantly higher in women $\left(24.6 \mathrm{~kg} / \mathrm{m}^{2}\right)$ than in men $\left(22.6 \mathrm{~kg} / \mathrm{m}^{2}\right)$; whereas the median value of $\mathrm{NC}$ was significantly higher in men $(37.8 \mathrm{~cm})$ than in women $(33.3 \mathrm{~cm})(\mathrm{p}<$ 0.001). In both men and women, NC was positively correlated with TC, LDL, TG and WC. Additionally, FPG and HDL were positively correlated with NC significantly. Moreover, there was a significant positive correlation between NECK and FPG but a significant negative correlation between NECK and HDLc among women. TG in men, raised TC, LDL and WC were found to be significantly associated with neck circumference with ORs 1.25 (95\% CI: 1.08, 1.44), 0.67 (95\% CI: $0.53,0.85), 1.13$ (95\% CI: $1.02,1.26), 1.27(95 \% \mathrm{CI}: 1.12,1.45)$ in men versus 1.18 (95\% CI: $1.03,1.36)$ in women, $1.19(95 \% \mathrm{CI}: 1.06,1.34)$ in men versus 1.21 (95\% CI: $1.06,1.38)$ in women and 1.18 (95\% CI: $1.06,1.36)$ in men versus 1.43 (95\% CI: $1.23,1.66)$ in women, respectively. Cut-off points for NC to identify CMRF were between 37.5 and $38 \mathrm{~cm}$ in men, 32.5 and $33 \mathrm{~cm}$ in women. Conclusion: NC is associated with CMRF, and could be a useful and accurate tool to identify high risk participants.
\end{abstract}

Keywords: Neck Circumference, Cardiometabolic Risk, Kinshasa

\section{Introduction}

Cardiovascular disease (CVD) is the leading cause of disability adjusted life years worldwide, particularly in developing countries [1]. The global cardiovascular risk is the probability of suffering from a coronary event or stroke in a given period of time and in this sense it is an absolute risk, generally reported as percentage at 10 years. Usually risk functions are used derived from longitudinal studies of healthy people at baseline [2]. They consider some factors that are coherently linked with events in population analyses: among these there are some metabolic factors (total cholesterol, HDL cholesterol, fasting blood glucose), some biological factors (blood pressure) and some lifestyle factors (tobacco smoking), all modifiable beyond those nonmodifiable like age and gender. Upper-body subcutaneous 
adipose tissue, estimated by neck circumference (NC), is a unique fat depot that may confer additional risk for metabolic risk factors over generalized and central adiposity [3-6]. NC as an index for upper-body subcutaneous adipose tissue distribution has been evaluated in relation to cardiovascular risk factors, insulin resistance, and biochemical components of metabolic syndrome (MetS) [7-10]. However, epidemiological population-based studies on the clinical significance of $\mathrm{NC}$ in connection with $\mathrm{CMRF}$ in general people are lacking. The aim of this study was to investigate the association between NC and same traditional CMRF.

\section{Patients and Methods}

\subsection{Study Population}

This cross-sectional study was made in general and eligible population of Kinshasa. Data were collected between November and December 2019 in Congolese participants aged 20 to 80 years, who had lived in the community over 10 years. Participants who met any of the following criteria were excluded from the study: abdominal disease that might affect the distribution of fat; a known Diabetes mellitus (DM); goiter; history of using cortical steroids; current treatment with statins or glucocorticoid; Cushing's syndrome; and recent, substantial weight loss or weight gain.

\subsection{Sample Size}

The minimal random sample size was calculated using the following formula:

$$
n=\frac{(\mathrm{Z})^{2} x(p)(q)}{(\mathrm{d})^{2}}
$$

$\mathrm{Z}=$ parameter related to statistical risk of admitted error $=1.96$ for a $5 \%$ error risk

$\mathrm{q}=$ assumed proportion of the target population not having the problem $(q=1-p)$

$\mathrm{p}=$ expected prevalence for MetS from a recent known prevalence of absolute accuracy $=11 \%$ in Kinshasa Hiterland [11].

$\mathrm{d}=$ absolute accuracy $=5 \%$

$$
n=\frac{(1.96)^{2} x(0.11)(0.89)}{(0.05)^{2}}=150
$$

We add $25 \%$ possible loss which makes a minimal of 188 participants. A total of 400 participants were analyzed in this study.

\subsection{Ethical Consideration}

The study design protocol was approved by the Ethics Committee of Lomo University of Research. Written informed consents were obtained from all patients. All procedures were in accordance with the Helsinki Declaration of 1975 , as revised in 2008 .

\subsection{Clinical and Anthropometric Evaluation}

Waist circumference (WC) was measured using flexible tape between the highest lateral edge of the right and left Ilium. NC was measured in the middle of the neck between the mid-cervical spine and the mid-anterior neck at $0.5 \mathrm{~cm}$, so palpable, just below the laryngeal prominence. BMI was calculated as the weight in kilograms divided by the height in meters squared. WHR and WHtR were calculated as waist circumference divided by hip circumference and height, respectively. Blood pressure (BP) was measured 3 times in a sitting position after at least 15 minutes of rest using an electronic type blood pressure monitor (OMRON M3 IT). The average of 3 recorded systolic and diastolic BP values was used in the analysis.

\subsection{Biochemical Measurements}

Peripheral venous blood samples were drawn after an overnight fast of at least $8 \mathrm{~h}$. The blood samples for the plasma glucose test were collected into vacuum tubes with the anticoagulant sodium fluoride and centrifuged within $1 \mathrm{~h}$ after collection. Plasma fasting concentrations of Glucose (FPG), Total Cholesterol (TC), Triglycerides (TG), highdensity lipoprotein cholesterol (HDL) and Uric acid were measured using the standard procedure using a COBAS C111 (Roche France). Insulin was detected by the chemiluminescence method (Abbott 12000 SR, USA). Then, insulin resistance was estimated by the homeostatic model assessment (HOMA-IR) index: [FI $(\mathrm{mIU} / \mathrm{L}) \times$ FPG $(\mathrm{mmol} / \mathrm{L})] / 22.5$ [12].

\subsection{Definitions of Variables}

High blood pressure (BP) was defined as BP $130 / 85 \mathrm{~mm}$ $\mathrm{Hg}$ or use of antihypertensive medication; low plasma highdensity lipoprotein cholesterol (HDL-C) cholesterol as HDL-C $<1.0 \mathrm{mmol} / \mathrm{L}$ in men and HDL-C $<1.3 \mathrm{mmol} / \mathrm{L}$ in women. Raised plasma triglycerides (TG) was defined as $\mathrm{TG}<1.7$ $\mathrm{mmol} / \mathrm{L}$ and raised fasting plasma glucose (FPG) as $\mathrm{FG}>5.6$ $\mathrm{mmol} / \mathrm{L} 1$. Increased waist circumference was defined as $\mathrm{WC}>$ $81 \mathrm{~cm}$ in both males and females, raised total plasma cholesterol (TC) as TC $>5.0 \mathrm{mmol} / \mathrm{L}$; and raised plasma lowdensity lipoprotein cholesterol (LDL-C) as LDL-C $>3.0$ $\mathrm{mmol} / \mathrm{L}$.

\subsection{Statistical Analyzes}

Data analyses were performed with the software package SPSS Statistics, Version 21 (IBM Corporation, Armonk, NY, USA). Normally distributed data were expressed as the means $\pm \mathrm{SD}$, whereas continuous variables with a skewed distribution were summarized as the median with interquartiles range. To compare the differences between groups, one-way analysis of variance (ANOVA) was used for continuous variables with a Gaussian distribution, and the Mann-Whitney U test was used for variables with a skewed distribution. Spearman's correlation coefficient was employed to test the correlations between different variables. The association of NC (independent variable) with cardiometabolic risk factors (dependent variables) was assessed by logistic regression. The receiver operating 
characteristics (ROC) curve analysis had allowed determining the optimal threshold of $\mathrm{NC}$ to detect the presence of cardiometabolic risk factors. A $P$ value $<0.05$ was considered statistically significant.

\section{Results}

\subsection{General Characteristics of Participants}

Table 1 shows the general characteristics of study population stratified by sex. Our survey included 400 participants (200 men and 200 women) with the mean age of
$55.4 \pm 12.0$ years and the mean WC of $79.8 \pm 12.0 \mathrm{~cm}$. The median value of BMI was significantly higher in women $(24.6$ $\left.\mathrm{kg} / \mathrm{m}^{2}\right)$ than in men $\left(22.6 \mathrm{~kg} / \mathrm{m}^{2}\right)(\mathrm{p}=0.002)$, whereas the median value of $\mathrm{NC}$ was significantly higher in men $(37.8 \mathrm{~cm})$ than in women $(33.3 \mathrm{~cm})(\mathrm{p}<0.001)$. WHtR, TC, LDL and TG were significantly higher in women. The majority of participants $(83.8 \%)$ had low HDL. Increased WC, high BP, raised FPG, TC, TG and LDL were observed respectively at $37.3 \%, 34 \%, 62.8 \%, 21.3 \%, 37.5 \%$ and $31.3 \%$ in study population.

Table 1. General characteristics of study population by sex.

\begin{tabular}{|c|c|c|c|c|c|c|}
\hline Variable & All $(n=400)$ & \multicolumn{2}{|c|}{ Men $(n=200)$} & \multicolumn{2}{|c|}{ Women $(n=200)$} & $p$ \\
\hline Age, year & $55.4 \pm 12.0$ & \multicolumn{2}{|c|}{$55.9 \pm 12.2$} & \multicolumn{2}{|c|}{$55.0 \pm 11.9$} & 0.334 \\
\hline BMI, $\mathrm{kg} / \mathrm{m}^{2}$ & $23.4(19.6-27.0)$ & \multicolumn{2}{|c|}{$22.6(19.3-26.0)$} & \multicolumn{2}{|c|}{$24.6(21-28)$} & 0.002 \\
\hline $\mathrm{WC}, \mathrm{cm}$ & $79.8 \pm 12.0$ & \multicolumn{2}{|c|}{$78.9 \pm 11.5$} & \multicolumn{2}{|c|}{$80.8 \pm 12.4$} & 0.131 \\
\hline $\mathrm{NC}, \mathrm{cm}$ & $36.0(33.1-38.1)$ & \multicolumn{2}{|c|}{$37.8(36.3-39.4)$} & \multicolumn{2}{|c|}{$33.3(31.9-34.6)$} & $>0.001$ \\
\hline WHtR & $0.48(0.42-0.56)$ & \multicolumn{2}{|c|}{$0.47(0.41-0.54)$} & \multicolumn{2}{|c|}{$0.48(0.44-0.57)$} & 0.026 \\
\hline $\mathrm{SBP}, \mathrm{mmHg}$ & $128.6 \pm 28.1$ & \multicolumn{2}{|c|}{$129.3 \pm 28.1$} & \multicolumn{2}{|c|}{$127.9 \pm 28.3$} & 0.440 \\
\hline DBP, $\mathrm{mmHg}$ & $73.6 \pm 13.8$ & \multicolumn{2}{|c|}{$73.8 \pm 13.5$} & \multicolumn{2}{|c|}{$73.3 \pm 14.2$} & 0.999 \\
\hline $\mathrm{FPG}, \mathrm{mmol} / \mathrm{L}$ & $6.3(5.1-8.1)$ & \multicolumn{2}{|c|}{$6.2(5.0-9.0)$} & \multicolumn{2}{|c|}{$6.2(5.4-8.0)$} & 0.702 \\
\hline $\mathrm{HbAlc}, \%$ & $6.6(4.0-11.4)$ & \multicolumn{2}{|c|}{$6.5(4.0-11.0)$} & \multicolumn{2}{|c|}{$6.8(4.0-12.0)$} & 0.834 \\
\hline HOMA-IR & $4.95(3.03-9.12)$ & \multicolumn{2}{|c|}{$4.41(2.67-8.70)$} & \multicolumn{2}{|c|}{$5.33(3.15-9.50)$} & 0.070 \\
\hline $\mathrm{TC}, \mathrm{mmol} / \mathrm{L}$ & $3.5(3.1-4.8)$ & \multicolumn{2}{|c|}{$3.4(2.8-4.2)$} & \multicolumn{2}{|c|}{$3.7(3.2-5.2)$} & 0.001 \\
\hline $\mathrm{HDL}, \mathrm{mmol} / \mathrm{L}$ & $0.52(0.31-0.88)$ & \multicolumn{2}{|c|}{$0.53(0.31-0.91)$} & \multicolumn{2}{|c|}{$0.48(0.31-0.83)$} & 0.398 \\
\hline $\mathrm{LDL}, \mathrm{mmol} / \mathrm{L}$ & $2.3(1.5-3.3)$ & \multicolumn{2}{|c|}{$2.2(1.2-3.1)$} & \multicolumn{2}{|c|}{$2.5(1.7-4.0)$} & 0.004 \\
\hline $\mathrm{TG}, \mathrm{mmol} / \mathrm{L}$ & $1.4(0.9-2.0)$ & \multicolumn{2}{|c|}{$1.01(0.82-1.88)$} & \multicolumn{2}{|c|}{$1.60(0.95-2.11)$} & $>0.001$ \\
\hline TG/HDL & $2.7(1.1-5.7)$ & 2.4 & $-4.9)$ & $3.1(1$ & & 0.009 \\
\hline $\mathrm{BP}>130 / 85 \mathrm{mmHg}$ & $136(34.0)$ & 70( & & $66(16$ & & 0.673 \\
\hline $\mathrm{FPG}>5.6 \mathrm{mmol} / \mathrm{L}$ & $251(62.8)$ & 128 & & 123 & & 0.605 \\
\hline $\mathrm{TC}>5.0 \mathrm{mmol} / \mathrm{L}$ & $85(21.3)$ & 32( & & $53(13$ & & 0.009 \\
\hline Low HDL mmol/L & $335(83.8)$ & 161 & $.3)$ & $174(4$ & & 0.078 \\
\hline $\mathrm{LDL}>3 \mathrm{mmol} / \mathrm{L}$ & $125(31.3)$ & 52( & & $73(18$ & & 0.023 \\
\hline $\mathrm{TG}>1.7 \mathrm{mmol} / \mathrm{L}$ & $150(37.5)$ & 61( & & $89(22$ & & 0.004 \\
\hline $\mathrm{WC}>81 \mathrm{~cm}$ & $149(37.3)$ & 68( & & $81(20$ & & 0.179 \\
\hline & & & & $\mathrm{NC}$ & WC & WHtR \\
\hline 3.2. Correlation $\mathrm{E}$ & and Cardiom & & SBP & 0.137 & $0.260^{\mathrm{b}}$ & 0.127 \\
\hline Factors & & & DBP & -0.049 & $0.148^{\mathrm{a}}$ & 0.060 \\
\hline & & & FPG & $0.295^{\mathrm{b}}$ & $0.189^{\mathrm{b}}$ & 0.135 \\
\hline Table 2 demor & correlation be & with & $\mathrm{TC}$ & $0.165^{\mathrm{a}}$ & $0.201^{\mathrm{b}}$ & 0.069 \\
\hline same cardiometab & actors. In both $\mathrm{n}$ & nen, & HDL & $-0.231^{\mathrm{b}}$ & $-0.141^{\mathrm{a}}$ & -0.071 \\
\hline $\mathrm{NC}$ was positivel & d with TC, LD & $\mathrm{WC}$. & LDL & $0.198^{b}$ & $0.229^{\mathrm{b}}$ & 0.090 \\
\hline Additionally in & was nositively & & TG & $0.244^{\mathrm{b}}$ & 0.080 & 0.014 \\
\hline
\end{tabular}
FPG and negatively with HDL significantly. Compared with $\mathrm{WC}$ and WHtR, those correlations were advanced, excepted $\mathrm{TC}$ and LDL in women.

Table 2. Correlation analysis between NC, WC and WHtR with CMRF factors by sex.

\begin{tabular}{llll}
\hline & NC & WC & WHtR \\
\hline Men $(\mathrm{n}=200)$ & & & \\
SBP & 0.018 & $0.209^{\mathrm{b}}$ & $0.182^{\mathrm{a}}$ \\
DBP & -0.038 & 0.040 & 0.104 \\
FPG & 0.115 & $0.192^{\mathrm{b}}$ & $0.163^{\mathrm{a}}$ \\
TC & $0.291^{\mathrm{b}}$ & $0.249^{\mathrm{b}}$ & $0.166^{\mathrm{a}}$ \\
HDL & -0.012 & -0.038 & -0.079 \\
LDL & $0.242^{\mathrm{b}}$ & $0.221^{\mathrm{b}}$ & $0.175^{\mathrm{a}}$ \\
TG & $0.165^{\mathrm{a}}$ & 0.127 & 0.061 \\
Women $(\mathrm{n}=200)$ & & & \\
\hline
\end{tabular}

a: $\mathrm{p}<0.05, \mathrm{~b}: \mathrm{p}<0.01$.

\subsection{Association Between NC with Cardiometabolic Risk Factors}

Hyperglycemia and low HDL in women, TG in men, raised TC, LDL and $\mathrm{WC}$ were found to be significantly associated with neck circumference from logistic regression analysis with ORs 1.25 (95\% CI: 1.08, 1.44), 0.67 (95\% CI: $0.53,0.85), 1.13$ (95\% CI: 1.02, 1.26), 1.27 (95\% CI: 1.12 , $1.45)$ in men versus 1.18 (95\% CI: $1.03,1.36)$ in women, 1.19 (95\% CI: $1.06,1.34)$ in men versus 1.21 (95\% CI: 1.06 , $1.38)$ in women and 1.18 (95\% CI: $1.06,1.36)$ in men versus 1.43 (95\% CI: $1.23,1.66)$ in women, respectively (see Table 3 , Figure 1, Figure 2). 
Table 3. Regression analysis to identify cardiometabolic risk factors associated with neck circumference (continuous independent variable) compared between men and women.

\begin{tabular}{|c|c|c|c|c|c|}
\hline \multirow{2}{*}{ Dependent Variables } & \multirow{2}{*}{ NC (tertiles) } & \multicolumn{2}{|l|}{ Men } & \multicolumn{2}{|l|}{ Women } \\
\hline & & OR (95\% IC) & $p$ & OR (95\% IC) & $p$ \\
\hline \multirow{4}{*}{$\mathrm{BP}>130 / 85 \mathrm{mmHg}$} & $\mathrm{T} 1$ & 1 & - & 1 & - \\
\hline & $\mathrm{T} 2$ & $2.06(1.00-4.25)$ & 0.050 & $1.38(0.65-2.93)$ & 0.401 \\
\hline & $\mathrm{T} 3$ & $1.28(0.61-2.69)$ & 0.521 & $1.86(0.89-3.88)$ & 0.099 \\
\hline & All & $1.04(0.94-1.16)$ & 0.450 & $1.13(0.99-1.30)$ & 0.064 \\
\hline \multirow{4}{*}{$\mathrm{FPG}>5.6 \mathrm{mmol} / \mathrm{L}$} & $\mathrm{T} 1$ & 1 & - & 1 & - \\
\hline & $\mathrm{T} 2$ & $0.83(0.41-1.68)$ & 0.598 & $2.09(1.04-4.20)$ & 0.038 \\
\hline & $\mathrm{T} 3$ & $0.92(0.45-1.88)$ & 0.812 & $3.24(1.57-6.70)$ & 0.002 \\
\hline & All & $1.03(0.93-1.15)$ & 0.533 & $1.25(1.08-1.44)$ & 0.002 \\
\hline \multirow{4}{*}{$\mathrm{TC}>5 \mathrm{mmol} / \mathrm{L}$} & $\mathrm{T} 1$ & 1 & - & 1 & - \\
\hline & $\mathrm{T} 2$ & $1.83(0.58-5.79)$ & 0.303 & $2.02(0.85-4.79)$ & 0.110 \\
\hline & $\mathrm{T} 3$ & $4.41(1.53-12.73)$ & 0.006 & $3.27(1.42-7.55)$ & 0.005 \\
\hline & All & $1.27(1.12-1.45)$ & $<0.001$ & $1.18(1.03-1.36)$ & 0.021 \\
\hline \multirow{4}{*}{ Low HDL } & $\mathrm{T} 1$ & 1 & - & 1 & - \\
\hline & $\mathrm{T} 2$ & $1.77(0.76-4.11)$ & 0.186 & $0.24(0.09-0.64)$ & 0.005 \\
\hline & $\mathrm{T} 3$ & $0.86(0.34-2.19)$ & 0.754 & $0.04(0.01-0.28)$ & 0.001 \\
\hline & All & $0.95(0.83-1.08)$ & 0.432 & $0.67(0.53-0.85)$ & 0.001 \\
\hline \multirow{4}{*}{$\mathrm{LDL}>3 \mathrm{mmol} / \mathrm{L}$} & $\mathrm{T} 1$ & 1 & - & 1 & - \\
\hline & $\mathrm{T} 2$ & $1.98(0.84-4.69)$ & 0.121 & $1.86(0.87-3.99)$ & 0.111 \\
\hline & T3 & $3.07(1.33-7.10)$ & 0.009 & $3.33(1.58-7.04)$ & 0.002 \\
\hline & All & $1.19(1.06-1.34)$ & 0.003 & $1.21(1.06-1.38)$ & 0.005 \\
\hline \multirow{3}{*}{$\mathrm{TG}>1.7 \mathrm{mmol} / \mathrm{L}$} & $\mathrm{T} 1$ & 1 & - & 1 & - \\
\hline & $\mathrm{T} 2$ & $1.18(0.53-2.52)$ & 0.675 & $1.79(0.89-3.61)$ & 0.105 \\
\hline & $\mathrm{T} 3$ & $1.58(0.75-3.32)$ & 0.323 & $2.07(1.03-4.18)$ & 0.041 \\
\hline \multirow{5}{*}{$\mathrm{WC}>81 \mathrm{~cm}$} & All & $1.13(1.02-1.26)$ & 0.024 & $1.14(1.00-1.29)$ & 0.050 \\
\hline & $\mathrm{T} 1$ & 1 & - & 1 & - \\
\hline & $\mathrm{T} 2$ & $1.89(0.84-3.90)$ & 0.125 & $3.80(1.73-8.37)$ & 0.001 \\
\hline & $\mathrm{T} 3$ & $2.54(1.20-5.40)$ & 0.015 & $5.60(2.54-12.31)$ & $<0.001$ \\
\hline & All & $1.18(1.06-1.32)$ & 0.003 & $1.43(1.23-1.66)$ & $>0.001$ \\
\hline
\end{tabular}

$\mathrm{T} 1<36.5 \mathrm{~cm}, \mathrm{~T} 236.5-39 \mathrm{~cm}, \mathrm{~T} 3>39 \mathrm{~cm}$ in men; T1 $<32 \mathrm{~cm}, \mathrm{~T} 232-34 \mathrm{~cm}, \mathrm{~T} 3>34 \mathrm{~cm}$ in women; Low HDL: $<1 \mathrm{mmol} / \mathrm{L}$ in men, $<1.3 \mathrm{mmol} / \mathrm{L}$ in women.

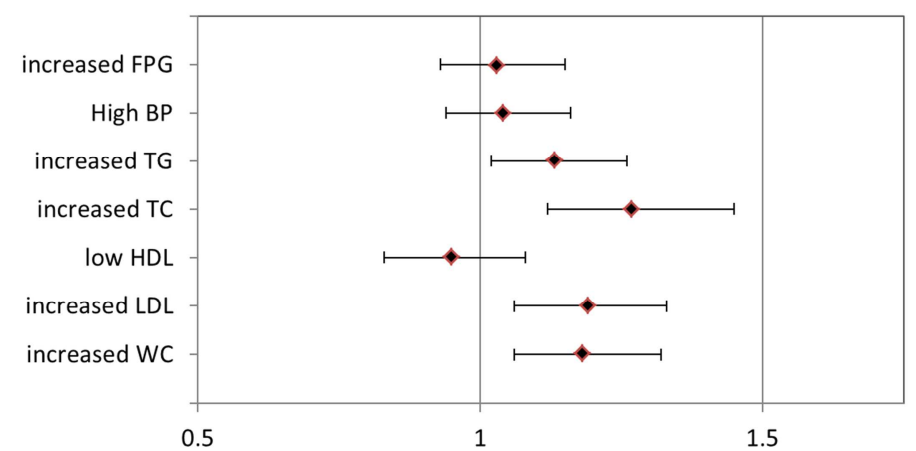

Figure 1. Odds ratio for increased FPG, high BP, raised TG, TC, LDL, WC and low HDL by neck circumference in men.

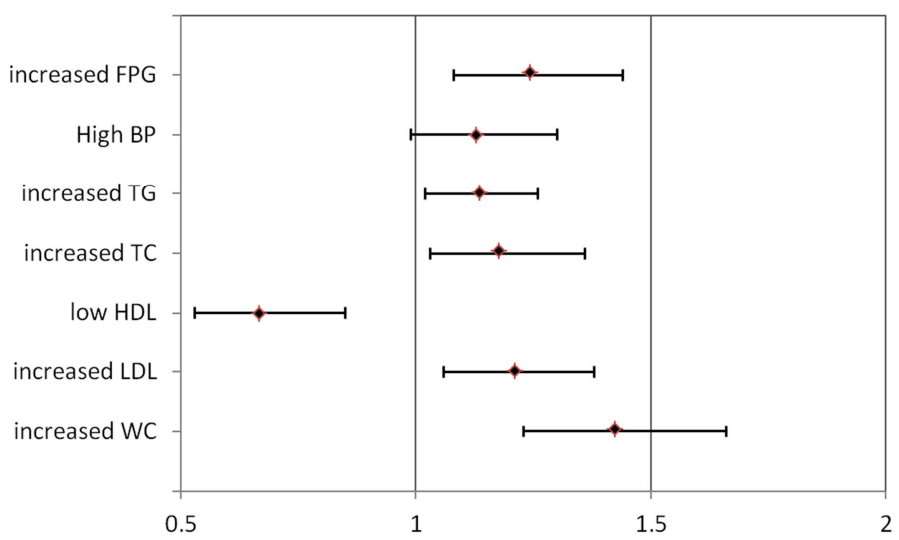

Figure 2. Odds ratio for increased FPG, high $B P$, raised $T G, T C, L D L, W C$ and low $H D L$ by neck circumference in women. 


\subsection{NC Cut-off Values for Cardiometabolic Risk Factors}

We determined the NC cut-off levels by relating them to risk factors. Tables 4 and 5 show the sensitivity and specificity for each $\mathrm{NC}$ level for the detection of raised blood pressure, hyperglycemia and dyslipidemia in men and women. Cut-off points for NC where sensitivity approximates specificity for each risk factor are between 37.5 and $38 \mathrm{~cm}$ in men, between 32.5 and $33 \mathrm{~cm}$ in women.

Table 4. Sensitivity and specificity for neck circumference cut-off points for cardiometabolic risk factors in men.

\begin{tabular}{|c|c|c|c|c|c|c|c|c|}
\hline \multirow{2}{*}{ NC cut-off $(\mathrm{cm})$} & \multicolumn{2}{|c|}{$\mathrm{BP}>130 / 85 \mathrm{mmHg}$} & \multicolumn{2}{|c|}{ FPG $>5.6 \mathrm{mmol} / \mathrm{L}$} & \multicolumn{2}{|c|}{$\mathrm{TG}>1.7 \mathrm{mmol} / \mathrm{L}$} & \multicolumn{2}{|c|}{$\mathrm{TC}>5.0 \mathrm{mmol} / \mathrm{L}$} \\
\hline & Se $(\%)$ & Sp (\%) & Se $(\%)$ & Sp (\%) & $\operatorname{Se}(\%)$ & Sp (\%) & $\operatorname{Se}(\%)$ & Sp (\%) \\
\hline 36 & 88.6 & 13.8 & 83.6 & 6.9 & 88.5 & 13.7 & 96.9 & 14.9 \\
\hline 37 & 71.4 & 42.3 & 61.7 & 36.1 & 68.9 & 40.3 & 81.3 & 41.1 \\
\hline 37.5 & 60.0 & 46.2 & 57.0 & 45.8 & 68.9 & 49.6 & 78.1 & 48.2 \\
\hline 37.7 & 58.6 & 51.5 & 54.4 & 52.8 & 65.6 & 54.0 & 78.1 & 53.8 \\
\hline 38.5 & 38.6 & 58.5 & 43.8 & 66.7 & 47.5 & 63.3 & 62.5 & 64.3 \\
\hline 39 & 22.9 & 70.0 & 27.3 & 72.2 & 29.5 & 73.4 & 46.9 & 76.2 \\
\hline 39.5 & 20.0 & 76.9 & 23.4 & 80.6 & 27.9 & 80.6 & 43.8 & 82.1 \\
\hline 40 & 15.7 & 81.5 & 18.8 & 84.7 & 26.2 & 86.3 & 34.4 & 85.7 \\
\hline 41 & 14.3 & 90.8 & 12.5 & 91.7 & 18.0 & 92.1 & 21.9 & 91.1 \\
\hline AUC & \multicolumn{2}{|l|}{0.526} & \multicolumn{2}{|l|}{0.511} & \multicolumn{2}{|l|}{0.585} & \multicolumn{2}{|l|}{0.695} \\
\hline$p$ value & \multicolumn{2}{|l|}{0.542} & \multicolumn{2}{|c|}{0.796} & \multicolumn{2}{|c|}{0.055} & \multicolumn{2}{|l|}{$>0.001$} \\
\hline
\end{tabular}

Table 4. Continued.

\begin{tabular}{|c|c|c|c|c|c|c|}
\hline \multirow{2}{*}{ NC cut-off $(\mathrm{cm})$} & \multicolumn{2}{|c|}{$\mathrm{HDL}>1 \mathrm{mmol} / \mathrm{L}$} & \multicolumn{2}{|c|}{$\mathrm{LDL}>3 \mathrm{mmol} / \mathrm{L}$} & \multicolumn{2}{|c|}{$W C>81 \mathrm{~cm}$} \\
\hline & Se $(\%)$ & Sp (\%) & Se $(\%)$ & Sp (\%) & Se $(\%)$ & Sp (\%) \\
\hline 36 & 84.5 & 2.6 & 96.2 & 16.2 & 95.6 & 17.4 \\
\hline 37 & 62.1 & 35.9 & 78.8 & 43.2 & 76.5 & 44.7 \\
\hline 37.5 & 57.8 & 51.3 & 75.0 & 50.7 & 72.1 & 52.3 \\
\hline 37.7 & 55.3 & 61.5 & 73.1 & 55.4 & 67.6 & 56.1 \\
\hline 38.5 & 44.1 & 74.4 & 55.8 & 64.9 & 51.5 & 65.2 \\
\hline 39 & 28.0 & 74.4 & 38.5 & 76.4 & 36.8 & 77.3 \\
\hline 39.5 & 23.6 & 84.6 & 32.7 & 81.8 & 30.9 & 82.6 \\
\hline 40 & 18.6 & 87.2 & 25.0 & 85.1 & 27.9 & 87.9 \\
\hline 41 & 12.4 & 94.9 & 13.5 & 89.9 & 16.2 & 90.9 \\
\hline AUC & \multicolumn{2}{|l|}{0.529} & \multicolumn{2}{|l|}{0.652} & \multicolumn{2}{|l|}{0.635} \\
\hline$p$ value & \multicolumn{2}{|l|}{0.577} & \multicolumn{2}{|l|}{0.001} & \multicolumn{2}{|l|}{0.002} \\
\hline
\end{tabular}

Table 5. Sensitivity and specificity for neck circumference cut-off points for cardiometabolic risk factors in women.

\begin{tabular}{|c|c|c|c|c|c|c|}
\hline \multirow{2}{*}{ NC cut - off $(\mathrm{cm})$} & \multicolumn{2}{|c|}{$\mathrm{BP}>130 / 85 \mathrm{mmHg}$} & \multicolumn{2}{|c|}{ FPG $>5.6 \mathrm{mmol} / \mathrm{L}$} & \multicolumn{2}{|c|}{$\mathrm{TG}>1.7 \mathrm{mmol} / \mathrm{L}$} \\
\hline & Se (\%) & Sp (\%) & Se (\%) & Sp (\%) & Se (\%) & Sp (\%) \\
\hline 31 & 93.9 & 6.1 & 94.3 & 7.8 & 95.5 & 8.1 \\
\hline 31.5 & 86.4 & 20.9 & 87.0 & 28.6 & 87.6 & 24.3 \\
\hline 32 & 78.8 & 33.6 & 78.0 & 41.6 & 77.5 & 35.1 \\
\hline 32.5 & 69.7 & 42.5 & 69.1 & 50.6 & 71.9 & 47.7 \\
\hline 33 & 59.1 & 47.8 & 61.8 & 57.1 & 66.3 & 55.0 \\
\hline 33.5 & 45.5 & 61.2 & 48.8 & 71.4 & 49.4 & 66.7 \\
\hline 34 & 40.9 & 69.4 & 40.7 & 76.6 & 39.3 & 70.3 \\
\hline 34.5 & 33.3 & 76.1 & 32.5 & 81.8 & 32.6 & 77.5 \\
\hline 35 & 30.3 & 82.1 & 26.8 & 85.7 & 25.8 & 81.1 \\
\hline 36 & 19.7 & 88.1 & 17.9 & 89.6 & 15.7 & 86.5 \\
\hline AUC & 0.572 & & 0.636 & & 0.600 & \\
\hline$(95 \% \mathrm{IC})$ & $(0.488-0.657)$ & & $(0.557-0.715)$ & & $(0.522-0.679)$ & \\
\hline$p$ value & 0.097 & & 0.001 & & 0.015 & \\
\hline
\end{tabular}


Table 5. Continued.

\begin{tabular}{|c|c|c|c|c|c|c|c|c|}
\hline \multirow{2}{*}{ NC cut-off $(\mathrm{cm})$} & \multicolumn{2}{|c|}{$\mathrm{TC}>5.0 \mathrm{mmol} / \mathrm{L}$} & \multicolumn{2}{|c|}{$\mathrm{HDL}>1.3 \mathrm{mmol} / \mathrm{L}$} & \multicolumn{2}{|c|}{$\mathrm{LDL}>3 \mathrm{mmol} / \mathrm{L}$} & \multicolumn{2}{|c|}{$W C>81 \mathrm{~cm}$} \\
\hline & Se $(\%)$ & Sp (\%) & $\operatorname{Se}(\%)$ & Sp (\%) & Se (\%) & Sp (\%) & $\mathrm{Se}(\%)$ & Sp (\%) \\
\hline 31 & 98.1 & 8.2 & 93.1 & 3.8 & 98.6 & 9.4 & 98.8 & 11.8 \\
\hline 31.5 & 96.2 & 24.0 & 82.8 & 30.8 & 93.2 & 25.2 & 93.8 & 27.7 \\
\hline 32 & 84.9 & 34.9 & 75.9 & 65.4 & 82.2 & 37.0 & 86.4 & 40.3 \\
\hline 32.5 & 79.2 & 45.2 & 67.2 & 80.8 & 78.1 & 48.0 & 82.7 & 52.9 \\
\hline 33 & 73.6 & 52.7 & 60.3 & 84.6 & 69.9 & 54.3 & 74.1 & 58.8 \\
\hline 33.5 & 54.7 & 65.1 & 46.0 & 92.3 & 54.8 & 66.9 & 54.3 & 68.1 \\
\hline 34 & 47.2 & 71.2 & 38.5 & 96.2 & 46.6 & 73.2 & 46.9 & 74.8 \\
\hline 34.5 & 34.0 & 75.3 & 31.0 & 100 & 34.2 & 77.2 & 39.5 & 81.5 \\
\hline 35 & 26.4 & 79.5 & 25.3 & 100 & 27.4 & 81.1 & 35.8 & 87.4 \\
\hline 36 & 15.1 & 85.6 & 16.7 & 100 & 15.1 & 85.8 & 24.7 & 91.6 \\
\hline AUC & \multicolumn{2}{|l|}{0.639} & \multicolumn{2}{|l|}{0.732} & \multicolumn{2}{|l|}{0.644} & \multicolumn{2}{|l|}{0.706} \\
\hline$(95 \% \mathrm{IC})$ & \multicolumn{2}{|c|}{$(0.556-0.716)$} & \multicolumn{2}{|c|}{$(0.672-0.827)$} & \multicolumn{2}{|c|}{$(0.568-0.721)$} & \multicolumn{2}{|c|}{$(0.634-0.777)$} \\
\hline
\end{tabular}

\section{Discussion}

Our study sought the optimal cut-off point of $\mathrm{NC}$ as indicator of CMRF in a large, representative sample of the Kinshasa adult population. The optimal cut-off point for WC was found to be $38 \mathrm{~cm}$ in men and $33 \mathrm{~cm}$ in women for identifying CMFR, and we suggest that these values could be used as the appropriate detection of individuals at high cardiometabolic risk in Kinshasa until long-term mortality data become available.

Similar to previously published data, we observed associations between traditional $\mathrm{CV}$ risk factors and increasing NC [10, 13-15]. Participants with largest NC had significantly higher waist circumference. Therefore, $\mathrm{NC}$ may be an important part of the routine risk assessment as it is associated with cardiometabolic risk factors and less intrusive to measure than waist circumference.

The limitation of our study is that the data are based on a cross-sectional survey that did not address the issue of $\mathrm{NC}$ and morbidity/mortality. Until long-term prospective studies are undertaken in various ethnic groups, cut-off points will have to be judged on studies such as ours.

The strength of the study lies in the fact that it is a large population-based study and representative of Kinshasa's adults. We describe, for the first time in Sub-Saharan region, the cross-sectional relationship of $\mathrm{NC}$ with cardiometabolic risk factors.

\section{Conclusion}

Our study suggests that a NC of $38 \mathrm{~cm}$ in men and $33 \mathrm{~cm}$ in women represents more appropriate cut-off points may potentially be beneficial in correctly identifying individuals at high cardiometabolic risk. Further research, particularly long-term, prospective, mortality studies, is urgently needed in Central Africa region to validate our findings.

\section{State of Current Knowledge on the Subject}

1. Cardiometabolic syndrome is a disease that is difficult to treat and diagnose;

2. Cardiometabolic syndrome is the basis of high morbidity and mortality;

3. Cardiometabolic syndrome is diagnosed at a late stage with a complication (obesity, hypertension, diabete millitus).

\section{Contribution of Our Study to Knowledge}

1. Poor knowledge of Cardiometabolic syndrome by healthcare providers in the DRC;

2. Poor diagnosis of disease to know its extent in the Congolese environment;

3. The cardiometabolic syndrome and the factors associated, previously unknown, with this work, currently available in DRC.

4. These results allow, in the clinical practice of healthcare providers, to improve the management of patients with Cardiometabolic syndrome. They also help to improve the monitoring of patients with Cardiometabolic syndrome.

\section{Conflict of Interest}

The authors declare no conflict of interest.

\section{Author's Contributions}

DMM and ANN designed and analyzed the statistical data for the study. M, VN and GLL contributed to the data collection. BLM, JBKL, EM supervised the study. All authors have read and approved the final and revised version of the manuscript. 


\section{Acknowledgements}

We thank all who participated in the study.

\section{References}

[1] Christopher JL, Murray CJL, Lopez AD. Measuring the global burden of disease. N Engl J Med. 2013; 369: 448-57. https ://doi.org/10.1056/NEJMra1201534.

[2] Dai Y, Wan X, Li X, Jin E, Li X. Neck circumference and future cardiovascular events in a high-risk population- $\mathrm{A}$ prospective cohort study. Lipids Health Dis. 2016; 5 (15): 46. https://doi.org/10.1186/s12944-016-0218-3.

[3] Grunfeld C, Rimland D, Gibert CL. Association of upper trunk and visceral adipose tissue volume with insulin resistance in control and HIV-infected subjects in the FRAM study. J Acquir Immune Defic Syndr 2007; 46: 283-290.

[4] Preis SR, Massaro JM, Hoffmann U, D'Agostino RB Sr, Levy D, Robins SJ, et al. Neck circumference as a novel measure of cardiometabolic risk: the Framingham Heart study. J Clin Endocrinol Metab. 2010; 95 (8): 3701-10.

[5] Stabe C, Vasques ACJ, Lima MMO, Tambascia MA, Pareja JC, Yamanaka A et al. Neck circumference as a simple tool for identifying the metabolic syndrome and insulin resistance: results from the Brazilian metabolic syndrome study. Clin Endocrinol. 2013; 78 (6): 874-81.

[6] Zhou JY, Ge H, Zhu MF, Wang LJ, Chen L, Tan YZ, et al. Neck circumference as an independent predictive contributor to cardio-metabolic syndrome. Cardiovasc Diabetol. 2013; 12 (76): 10-1186.

[7] Sjostrom CD, Håkangård AC, Lissner L, Sjostrom L. Body compartment and subcutaneous adipose tissue distribution risk factor patterns in obese subjects. Obes Res 1995; 3: 9-22.
[8] Onat A, Hergenc G, Yuksel H, Can G, Ayhan E, Kaya Z, et al. Neck circumference as a measure of central obesity: associations with metabolic syndrome and obstructive sleep apnea syndrome beyond waist circumference. Clin Nutr 2009; 28: 46-51.

[9] Laakso M, Matilainen V, Keinanen-Kiukaanniemi S. Association of neck circumference with insulin resistancerelated factors. Int J Obes Relat Metab Disord 2002; 26: 873875 .

[10] Ben-Noun LL, Laor A. Relationship between changes in neck circumference and cardiovascular risk factors. Exp Clin Cardiol 2006; 11: 14-20.

[11] Nasila Sungwacha J, Tyler J, Longo-Mbenza B, Lasi On'Kin JBK, Gombet T, Erasmus RT. Assessing clustering of metabolic syndrome components available at primary care for Bantu Africans using factor analysis in the general population. BMC Res Notes 2013; $6 \quad$ (1): 228. https://doi.org/10.1186/1756-0500-6-228.

[12] Galvin P, Wa rd G, Walters J, Pestell R, Koschmann M, Vaag $A$, et al. A simple method for quantitation of insulin sensitivity and insulin release from an intravenous glucose tolerance test. Diabet Med 9: 921-928, 1992.

[13] Joshipura K, Munoz-Torres F, Vergara J. Neck Circumference May Be a Better Alternative to Standard Anthropometric Measures. JDiabetes Res 2016; 2016: 6058916.

[14] Preis SR, Pencina MJ, D'Agostino Sr RB. Neck circumference and the development of cardiovascular disease risk factors in the Framingham Heart Study. Diabetes Care 2013; 36 (1): e3.

[15] Namazi N, Larijani B, Surkan PJ, Azadbakht L. The association of neck circumference with risk of metabolic syndrome and its components in adults: A systematic review and meta-analysis. Nutrition, Metabolism \& Cardiovascular Diseases (2018) 28, 657e674. 